\title{
Micropulse vs. continuous wave transscleral cyclophotocoagulation in neovascular glaucoma
}

\author{
MIHAIL ZEMBA ${ }^{1,2^{*}}$, OTILIA-MARIA DUMITRESCU ${ }^{1}$, FLORIN VAIDA $^{3}$, ELENA-ANDREEA DIMIRACHE ${ }^{1}$, \\ IULIA PISTOLEA $^{1}$, ALINA CRISTINA STAMATE ${ }^{4}$, MARIAN BURCEA $^{2 *}$, DANIEL CONSTANTIN BRANISTEANU ${ }^{5}$, \\ FLORIAN BALTA $^{2 *}$ and ILEANA RAMONA BARAC ${ }^{2}$ \\ ${ }^{1}$ Department of Ophthalmology, 'Dr. Carol Davila' Central Military Emergency University Hospital, 01082 Bucharest; \\ ${ }^{2}$ Department of Ophthalmology, 'Carol Davila' University of Medicine and Pharmacy, 050474 Bucharest, Romania; \\ ${ }^{3}$ Division of Biostatistics and Bioinformatics, University of California, San Diego, CA 92093, USA; \\ ${ }^{4}$ Department of Ophthalmology, Arena Med Clinic, 022117 Bucharest; ${ }^{5}$ Department of Ophthalmology, \\ ‘Grigore T. Popa' University of Medicine and Pharmacy, 700115 Iasi, Romania
}

Received August 17, 2021; Accepted September 16, 2021

DOI: 10.3892/etm.2022.11207

\begin{abstract}
Neovascular glaucoma (NVG) is a refractory form of glaucoma, associated with important morbidity, for which no consensus exists regarding the optimal choice of therapy. The primary aim of our study was to compare the performances of micropulse transscleral cyclophotocoagulation (MP-TSCPC) and continuous wave transscleral cyclophotocoagulation (CW-TSCPC) in the treatment of neovascular glaucoma (NVG). A total of 24 eyes for MP-TSCPC and 22 eyes for CW-TSCPC, all with NVG were included. The procedures were performed using either the Iridex Cyclo G6 (IRIDEX Laser System), the MP3, or the G-Probe devices. Intraocular pressure (IOP), visual acuity (VA), the mean number of antiglaucoma medications, and postoperative complications were monitored. The minimum follow-up was 12 months. The success rate at 12 months was $54.5 \%$ in the CW-TSCPC group and $33.3 \%$ in the MP-TSCPC group. The mean IOP at baseline was $35.82 \mathrm{~mm} \mathrm{Hg}$ for CW-TSCPC and $34.71 \mathrm{~mm} \mathrm{Hg}$ for
\end{abstract}

Correspondence to: Dr Daniel Constantin Branisteanu, Department of Ophthalmology, 'Grigore T. Popa' University of Medicine and Pharmacy, 16 University Street, 700115 Iasi, Romania E-mail: daniel.branisteanu@umfiasi.ro

*Contributed equally

Abbreviations: NVG, neovascular glaucoma; IOP, intraocular pressure; TS-CPC, transscleral cyclophotocoagulation; CW-TSCPC, continuous wave transscleral cyclophotocoagulation; MP-TSCPC, micropulse transscleral cyclophotocoagulation; BCVA, best-corrected visual acuity; $\mathrm{CF}$, counting fingers; HM, hand movements; LP, light perception

Key words: neovascular glaucoma, continuous-wave transscleral cyclophotocoagulation, micropulse transscleral cyclophotocoagulation, intraocular pressure, complications
MP-TSCPC. The change from baseline in IOP at 12 months was $11.95 \mathrm{~mm} \mathrm{Hg}$ in the CW-TSCPC group and $-8.04 \mathrm{~mm} \mathrm{Hg}$ in the MP-TSCPC group. There was a significant difference in the occurrence of serious complications (worsening of VA, hypotony, and phthisis bulbi) between the two methods, with CW-TSCPC associated with more important adverse effects $(\mathrm{P}=0.045)$. There was a decrease in the number of topical antiglaucoma medications in both groups: in the MP-TSCPC group from a mean number of 2.6 at baseline, to 1.7 at 3 months, followed by a slight increase to 2.1 at 12 months and in the CW-TSCPC group from 2.8 at baseline, to 1.4 at 3 months and 1.9 at 12 months. Our study concluded that both MP-TSCPC and CW-TSCPC could manage NVG, but, while CW-TSCPC revealed higher IOP control in the long term (which did not reach statistical significance), it also had a significantly lower safety profile.

\section{Introduction}

Neovascular glaucoma (NVG) is one of the most refractory forms of glaucoma, caused by various ocular and occasionally systemic conditions that produce retinal ischemia. NVG often appears as an end-stage disease, resulting in blindness, continuous pain, and eventually loss of the eye. In this stage, the objective of the treatment is to lower the intraocular pressure (IOP) to relieve the pain and preserve the globe (1). Numerous treatments have been attempted for lowering IOP in NVG, but no consensus exists regarding the most effective and safest procedure (2). Trabeculectomy with antimetabolites, aqueous shunt implantation, and cyclodestructive procedures are the main methods used to treat high IOP in NVG. For many years, a variety of methods resulting in cyclodestruction have been used to reduce the aqueous formation and, subsequently, the IOP. Non-penetrating and penetrating cyclodiathermy were introduced in the 1930s, cyclocryotherapy in the 1950s, and later high-intensity focused ultrasound, but all of these have been abandoned due to the high risk of devastating complications $(3,4)$. Cyclophotocoagulation is a form of cycloablation 
that focuses high-intensity laser energy at the level of ciliary epithelium, where it is absorbed by melanin and transformed into heat with a coagulative effect, resulting in the reduction of aqueous production and, consequently, in lowering of the IOP (5). Although numerous types of lasers have been used, diode lasers are currently considered to be the most appropriate. The diode laser emits a beam with a wavelength of $800-850 \mathrm{~nm}$, which is best absorbed by the melanin in the pigmentary epithelium, with less energy affecting the sclera. The energy delivery periods are quite long, 2-3 sec, and therefore, high energy is transferred to the ciliary stroma, with coagulative effects. Traditionally, transscleral cyclophotocoagulation (TSCPC) delivers laser energy in a continuous manner. Continuous wave transscleral cyclophotocoagulation (CW-TSCPC) is effective in lowering IOP, but has a risk of important complications such as a decrease in visual acuity (VA), hypotony, chronic uveitis, and phthisis bulbi (6-8). These complications are likely the result of damage to the surrounding tissues due to the spread of the thermal energy (6). Another technique, micropulse transscleral cyclophotocoagulation (MP-TSCPC), involves using a novel probe that delivers a series of short pulses of laser energy ('on') separated by rest periods ('off'). During the 'on' period the thermal energy acts on ciliary body epithelium, while during 'off' periods the adjacent structures are allowed to dissipate the heat, protecting them from the thermal effect. Therefore MP-TSCPC reduces the damage of the surrounding tissues and lowers the incidence of complications while preserving the IOP lowering activity $(6,9,10-12)$. The primary aim of the study was to compare the performance of MP-TSCPC vs. CW-TSCPC over 12 months post-intervention. The secondary aim of the study was to demonstrate the safety and efficacy of MP-TSCPC over 12 months post-intervention.

\section{Materials and methods}

Study design. A retrospective cohort study was performed including all patients with NVG that were treated with TSCPC between January 2017 and September 2019 at the Department of Ophthalmology of 'Dr. Carol Davila' Central Military Emergency University Hospital (Bucharest, Romania). While the study was not randomized, the treatment modality (MP vs. CW) was not based on medical considerations related to the case. All patients followed a fixed postoperative visit schedule: day 1 , day 7 , months $1,3,6,12$, and 15 . The primary time-point was 12 months post-intervention. The study was approved (approval no. 445/03.03.2021) by the Institutional Review Board of the hospital and followed the principles of the Declaration of Helsinki (2013).

Data of patients. Data were collected at baseline, before the TSCPC intervention on demographics of patients (age and sex), diagnosis including etiology of NVG, IOP, the number of glaucoma medications, including oral acetazolamide, best-corrected visual acuity (BCVA), anterior segment evaluation, and type of TSCPC used, MP or CW. A total of 51 eyes from 51 patients were treated, 27 with MP-TSCPC and 24 with CW-TSCPC. However, 5 were later excluded due to inadequate length of follow-up, and thus a remainder of 24 eyes for MP-TSCPC and 22 eyes for CW-TSCPC were included. The age was comparable between groups $(\mathrm{P}=0.45)$, with means of 55.6 years (range, 44-79) for MP-TSCPC and 58.1 years (range, 32-87) for CW-TSCPC. There were no differences between groups with regard to sex (for example, males, 54.2 and 59.1\% in the MP-TSCPC and the CW-TSCPC groups, respectively; $\mathrm{P}=0.97$ ), as demonstrated in Table I. At each follow-up visit, IOP, VA, antiglaucoma medications, complications, and the need for retreatment were recorded. IOP was assessed by Goldmann applanation tonometry, or by I-care rebound tonometry when Goldmann applanation tonometry was not accurate or possible. BCVA was at an extremely low level, counting fingers $(\mathrm{CF})$ or less. After the treatment, VA was divided into three groups, namely improved, unchanged, and worsened, compared with the baseline VA. A change in VA was defined as improved if it changed from light perception (LP) to perception of hand movements (HM) or improved, or from $\mathrm{HM}$ to $\mathrm{CF}$, unchanged if it remained the same or worsened, when there was a decline in VA, either from CF to HM or LP or from HM to LP.

Treatment. All procedures were performed in the operating room, under regional anesthesia; specifically, retrobulbar block with a mixture of $3 \mathrm{ml}$ of lidocaine $4 \%$ and $1 \mathrm{ml}$ of bupivacaine $1 \%$. Transillumination was used when the position of the ciliary body was in doubt (high myopes, multiple surgeries on the anterior pole). Methylcellulose was used as a coupling agent, to facilitate the movement of the probe tip and to increase the laser power transmission.

MP-TSCPC was performed with an MP P3 handpiece with the Iridex Cyclo G6 (IRIDEX Laser System). The power was set at $2000 \mathrm{~mW}$ and a duty cycle of $31.35 \%$ (micropulse 'on' for $0.5 \mathrm{msec}$ and 'off' for $1.1 \mathrm{msec}$ ). The probe was applied using firm, moderate pressure in a continuous, sweeping motion over the superior and inferior quadrants, $90 \mathrm{sec}$ for each hemiglobe. The 3 and 9 o'clock meridians, areas of scleral thinning, filtering blebs, and glaucoma drainage devices were avoided.

CW-TSCPC was performed with the G probe of Iridex Cyclo G6 (IRIDEX Laser System). A total of $75 \%$ of the eye circumference was treated. This usually required 6-7 applications in each quadrant, for a total of 20-21 shots. The initial power was $1,250 \mathrm{~mW}$ and the duration was $4 \mathrm{sec}$. The power of the laser was reduced by $200 \mathrm{~mW}$ if more than two 'pops' from disruption of the ciliary processes were heard.

Postoperatively dexamethasone $0.1 \%$ every $6 \mathrm{~h}$ and cyclopentolate $1 \%$ twice daily were indicated. Patients continued their antiglaucoma therapy after the procedure. Therapy was later adjusted with the oral acetazolamide according to the IOP values recorded during the follow-up visits.

Follow-up. Patients were examined the following day, at one week, at 1, 3, 6, 9, 12, and 15 months. A minimum of 12 months of follow-up was required for study inclusion.

Outcome measures. The primary outcome measure was a successful reduction of IOP: A 'favorable outcome' at any time-point was defined as postprocedural IOP $\leq 21 \mathrm{~mm} \mathrm{Hg}$ or IOP reduction from baseline of $\geq 30 \%$, with or without additional antiglaucoma medications. Hypotony was defined as an IOP of $<5 \mathrm{~mm} \mathrm{Hg}$ and was considered a failure of the treatment. 
Table I. Patient characteristics at baseline.

\begin{tabular}{lccc}
\hline Characteristics & $\begin{array}{c}\text { Micropulse transscleral } \\
\text { cyclophotocoagulation }\end{array}$ & $\begin{array}{c}\text { Continuous wave-transscleral } \\
\text { cyclophotocoagulation }\end{array}$ & P-value \\
\hline Age (years) & 55.6 (range, $44-79)$ & 58.1 (range, 32-87) & 0.30 \\
Sex & $13(54.2 \%)$ & $13(59.1 \%)$ & 0.74 \\
Male & $11(45.8 \%)$ & $9(40.9 \%)$ & 0.83 \\
Female & & & 0.69 \\
Etiology of neovascular glaucoma & $8(33.3 \%)$ & $8(36.3 \%)$ & 1.0 \\
Retinal vein occlusion & $9(37.5 \%)$ & $7(31.8 \%)$ & 0.41 \\
Diabetic retinopathy & $2(8.3 \%)$ & $1(4.5 \%)$ & 0.49 \\
Chronic uveitis & $2(8.3 \%)$ & $4(18.9 \%)$ & 1.0 \\
Chronic retinal detachment & 0 & $1(4.5 \%)$ & 1.0 \\
Retinal artery occlusion & $1(4.2 \%)$ & 0 & 0.57 \\
Radiotherapy induced & $2(8.3 \%)$ & $1(4.5 \%)$ & 1.0 \\
Carotid artery occlusive disease & & & 0.71 \\
Previous surgery & 10 & 11 & 0.70 \\
Trabeculectomy & 3 & 2 & 1.0 \\
Iridectomy/iridotomy & 10 & 8 & 0.49 \\
Posterior vitrectomy & 5 & 3 & 0.54 \\
Cataract surgery & 1 & 1 & 0.91 \\
Ahmed valve implantation & 0 & 1 & 7 \\
Penetrating keratoplasty & 8 & $0.95($ range, $0-5)$ & \\
Anti-VEGF injection & $1.33($ range, $0-5)$ & & \\
No previous surgery & 8 & & \\
\hline
\end{tabular}

Mean (range) or frequency (\%) reported.

Secondary outcome measures included the change in BCVA, the number of antiglaucoma medications, the necessity of oral acetazolamide, the complications, and the need for retreatment.

Statistical analysis. Baseline characteristics were summarized (number and proportions for categorical variables; mean and standard deviation for continuous variables), and compared between the two groups using Pearson's Chi-square test for categorical variables, and the Wilcoxon rank-sum test for continuous variables.

For the first study aim, the comparison of CW-TSCPC and MP-TSCPC procedures, the primary endpoint was the success of the intervention at 12 months. This was compared between the two arms using Pearson's Chi-squared test, and a $95 \%$ confidence interval $\left(\mathrm{CI}_{95}\right)$ of the difference in proportions of favorable outcomes between arms was reported. The proportion of favorable outcomes over time was compared between arms using longitudinal logistic regression using generalized estimating equations (GEE), which accounts for the correlation of outcomes within individuals. Moreover, the proportion of times with a favorable outcome over time between arms was compared using a one-degree of freedom Wald test within the GEE logistic regression model.

The secondary outcome was IOP, which was compared longitudinally between groups using a longitudinal general linear model (LGLM), including time treated as a factor, treatment, and their interaction. The model allows within-patient correlation and time-varying variance. The selection of best correlation structure (unstructured, constant, exponential and autoregressive of order 1), and time-constant vs. time-varying variance was made based on the Akaike information criterion. The comparison of the two treatment arms at month 12 used the Wald test of the LGLM. The overall comparison of IOP trajectories over time between the two arms used the Wald test for the interaction in the LGLM.

The important adverse events were considered worsening of VA, hypotony, and phthisis bulbi. The total of these three events was computed for each patient, and the rate of these events was compared between groups using a Poisson model, with a check for overdispersion (none was detected). Prevalence of individual important adverse events was compared between arms using Fisher's exact test.

All analyses were conducted using the $\mathrm{R}$ statistical language and the 'nlme' package version 3.1-148 was used. $\mathrm{P}<0.05$ was considered to indicate a statistically significant difference.

\section{Results}

Demographic and clinical characteristics. A total of 24 eyes from 24 patients with NVG treated using MP-TSCPC and 22 eyes from 22 patients with NVG treated using CW-TSCPC, 
were analyzed. The underlying causes of retinal ischemia are presented in Table I. Most eyes in both groups had undergone multiple surgeries. Only 8 eyes in the MP-TSCPC group and 7 eyes in the CW-TSCPC group had no previous surgery $(\mathrm{P}=0.91)$. The surgery types are listed in Table I.

Follow-up. The mean follow-up period was 15.5 \pm 2.1 months (range, 12-19) for the MP-TSCPC group; all the eyes reached 12 months and 19 eyes had 15 months of follow-up. For CW-TSCPC, the mean follow-up period was $15.9 \pm 2.3$ months (range, 12-21) with all the eyes reaching 12 months and 19 eyes reaching 15 months of follow-up.

Primary outcome. The percentage of favorable outcome (or successes) at month 12 was $54.5 \%$ in the CW-TSCPC group, (95\% CI, 34.1 to $73.5 \%$ ), and $33.3 \%$ in the MP-TSCPC group (95\% CI, 17.6 to $53.9 \%$ ). The odds ratio (OR) of favorable outcome, CW-TSCPC vs. MP-TSCPC was 2.40 [95\% CI $(0.73,7.92), \mathrm{P}=0.15]$. Averaged over the 12 months of follow-up, the percentage of favorable outcomes in the two arms was $64.6 \%$ [95\% CI $(46.9,79.1 \%]$ for the CW-TSCPC group, and $52.3 \%$ [95\% CI $(35.4,68.7 \%)$ for the MP-TSCPC group, with an OR of 1.67 [95\% CI $(0.69,4.04)$; $\mathrm{P}=0.25$ ]. Results are presented in Fig. 1 and Table II.

IOP. The change in IOP from baseline to 12 months was $-11.95 \mathrm{~mm} \mathrm{Hg}$ [95\% CI $(-17.77,-6.14) \mathrm{mm} \mathrm{Hg}$ ] in the CW-TSCPC group, and $-8.04 \mathrm{~mm} \mathrm{Hg}$ [95\% CI (-13.61, -2.48) $\mathrm{mm} \mathrm{Hg}$ ] in the MP-TSCPC group, for a difference of $-3.91 \mathrm{~mm} \mathrm{Hg}[95 \% \mathrm{CI}(-11.96,3.91) \mathrm{mm} \mathrm{Hg} ; \mathrm{P}=0.34]$. Averaged over the 12-month follow-up, the mean change in IOP from baseline was $-12.39 \mathrm{~mm} \mathrm{Hg}$ [95\% CI $(-16.22,-8.55) \mathrm{mm} \mathrm{Hg}]$ for the CW-TSCPC group, and $-10.04 \mathrm{~mm} \mathrm{Hg}$ [95\% CI (-13.71, -6.37) $\mathrm{mm} \mathrm{Hg}$ ] for the MP-TSCPC group, for a difference of $-2.34 \mathrm{~mm} \mathrm{Hg}$ [95\% CI $(-7.65,2.96) \mathrm{mm} \mathrm{Hg}$; $\mathrm{P}=0.39]$. The mean IOP at month 12 in the two groups was $23.86 \mathrm{~mm} \mathrm{Hg}$ [95\% CI $(18.07,29.66) \mathrm{mm} \mathrm{Hg}$ ] for the CW-TSCPC group, and $26.67 \mathrm{~mm} \mathrm{Hg}$, [95\% CI $(21.12,32.21) \mathrm{mm} \mathrm{Hg}$ ] for the MP-TSCPC group. Results are presented in Fig. 1 and Table II. The evolution of the IOP and of the success rate at follow-up for both MP-TSCPC and CW-TSCPC are revealed in Table III.

Important adverse events. The mean number of important adverse events over the primary 12-month follow-up was 0.636 [95\% CI $(0.358,1.030)$ for $\mathrm{CW}$, and 0.250 [95\% CI $(0.099,0.507)$ for the MP arm. The rate ratio was 2.55 [95\% CI $(1.02,7.19)]$ for CW-TSCPC vs. MP-TSCPC; $\mathrm{P}=0.045$. Results are presented in Table II.

Effects on antiglaucoma medication. For MP-TSCPC, the mean number of topical antiglaucoma medications at baseline was $2.6 \pm 1$; oral acetazolamide was initially used by 14 patients $(58.3 \%)$. The number of topical medications decreased during the first 3 months after treatment to $1.7 \pm 1.3$ and then started to increase, reaching $2.1 \pm 1.3$ at 12 months. The number of patients requiring oral acetazolamide exhibited a more pronounced decrease to only $25 \%$ at the end of the first month, $16.7 \%$ at the end of the 3rd and 6th months, and $20.8 \%$ at one year. The evolution of the antiglaucoma
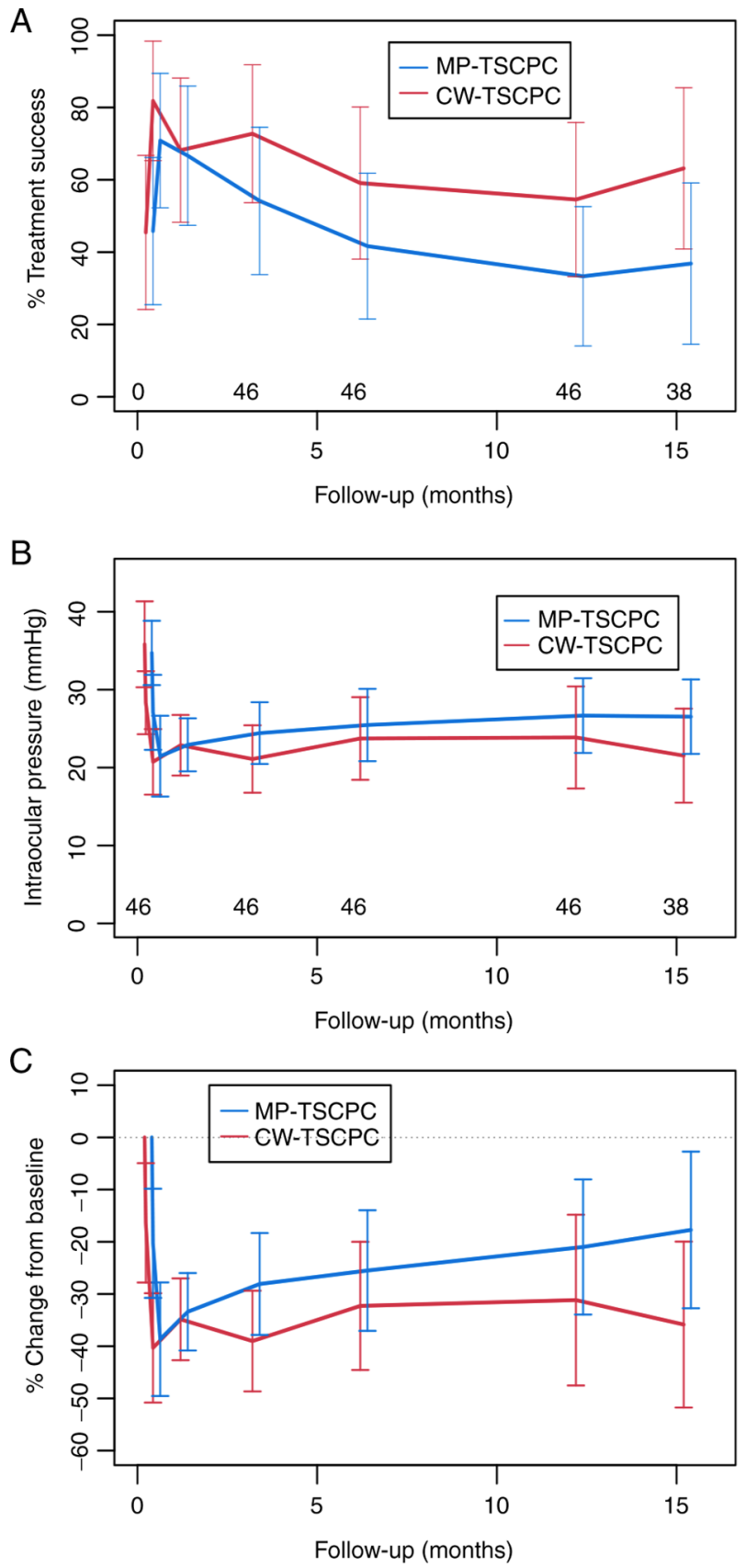

Figure 1. Comparative evolution of the success rate and IOP for MP-TSCPC and CW-TSCPC. Comparative evolution of (A) the success rate, (B) the IOP and $(\mathrm{C})$ the reduction of IOP expressed as a percentage (\%) from the baseline IOP for the two methods with time-points marked at baseline, week 1 , month 1, 3, 6, 12 and 15. IOP, intraocular pressure; CW-TSCPC, continuous wave transscleral cyclophotocoagulation; MP-TSCPC, micropulse transscleral cyclophotocoagulation.

medication after MP-TSCPC is presented in Table IV and Fig. 2.

For CW-TSCPC, the mean number of topical antiglaucoma drugs used at baseline was $2.8 \pm 0.8$ and 14 patients $(63.6 \%)$ used oral acetazolamide. The number of antiglaucoma drops was significantly reduced after the procedure, reaching the lowest level after 3 months $(1.4 \pm 1.4)$ and remained quite stable during the follow-up period. The same effect, but more pronounced, was achieved in the case of oral acetazolamide users. The number of patients using oral acetazolamide 
Table II. Clinical and adverse events outcomes in the MP-TSCPC (MP) and CW-TSCPC (CW) groups.

\begin{tabular}{|c|c|c|c|c|}
\hline Events & $\mathrm{CW}$ & MP & CW vs. MP & P-value \\
\hline A, Favorable outcome, proportion & $\%(95 \% \mathrm{CI})$ & $\%(95 \% \mathrm{CI})$ & OR $(95 \% \mathrm{CI})$ & \\
\hline Month 12 & $54.5(34.1,73.5)$ & $33.3(17.6,53.9)$ & $2.40(0.73,7.92)$ & 0.15 \\
\hline Average over day 1 to month 12 & $64.6(46.9,79.1)$ & $52.3(35.4,68.7)$ & $1.67(0.69,4.04)$ & 0.25 \\
\hline $\mathrm{B}$, IOP change from baseline & Mean $(95 \% \mathrm{CI}), \mathrm{mm} \mathrm{Hg}$ & Mean $(95 \% \mathrm{CI}), \mathrm{mm} \mathrm{Hg}$ & Difference (95\% CI) & $\mathrm{P}$-value \\
\hline Month 12 & $-11.95(-17.77,-6.14)$ & $-8.04(-13.61,-2.48)$ & $-3.91(-11.96,3.91)$ & 0.34 \\
\hline Average over day 1 to month 12 & $-12.39(-16.22,-8.55)$ & $-10.04(-13.71,-6.37)$ & $-2.34(-7.65,2.96)$ & 0.39 \\
\hline $\mathrm{C}, \mathrm{IOP}(\mathrm{mmHg})$ & Mean $(95 \% \mathrm{CI})$ & Mean $(95 \% \mathrm{CI})$ & Difference $(95 \% \mathrm{CI})$ & P-value \\
\hline Baseline & $35.82(30.90,40.74)$ & $34.71(30.00,39.42)$ & $1.11(-5.70,7.92)$ & 0.75 \\
\hline Month 12 & $23.86(18.07,29.66)$ & $26.67(21.12,32.21)$ & $2.80(-5.22,10.82)$ & 0.49 \\
\hline $\mathrm{D}$, Important adverse events & Rate $(95 \% \mathrm{CI})$ & Rate $(95 \% \mathrm{CI})$ & Rate ratio, $(95 \% \mathrm{CI})$ & P-value \\
\hline Day 0 to month 12 & $0.636(0.358,1.030)$ & $0.250(0.099,0.507)$ & $2.55(1.02,7.19)^{\mathrm{a}}$ & 0.045 \\
\hline
\end{tabular}

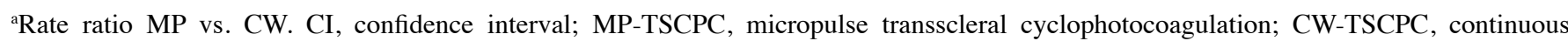
wave-transscleral cyclophotocoagulation. IOP, intraocular pressure.

Table III. Evolution of the IOP and of the success rate after MP-TSCPC and CW-TSCPC at different time-points.

\begin{tabular}{|c|c|c|c|c|c|c|c|}
\hline Procedure & Outcome & Baseline & 1 week & 1 month & 3 months & 6 months & 12 months \\
\hline \multirow[t]{3}{*}{ MP-TSCPC } & $\begin{array}{l}\text { Mean IOP } \\
(\mathrm{mm} \mathrm{Hg})\end{array}$ & $34.7 \pm 10.3$ & $21.4 \pm 12.9$ & $23.1 \pm 8.5$ & $24.3 \pm 9.9$ & $25.4 \pm 1.6$ & $26.7 \pm 12$ \\
\hline & $\begin{array}{l}\text { IOP reduction } \\
\text { (from baseline) }\end{array}$ & & $38.3 \%$ & $32.6 \%$ & $29.9 \%$ & $26.8 \%$ & $23.0 \%$ \\
\hline & Success rate & & $70.8 \%$ & $66.6 \%$ & $58.3 \%$ & $41.6 \%$ & $29.1 \%$ \\
\hline \multirow[t]{3}{*}{ CW-TSCPC } & $\begin{array}{l}\text { Mean IOP } \\
(\mathrm{mm} \mathrm{Hg})\end{array}$ & $36.0 \pm 13.2$ & $20.7 \pm 10$ & $22.8 \pm 9.3$ & $21.6 \pm 10.3$ & $23.7 \pm 12.7$ & $23.9 \pm 15.6$ \\
\hline & $\begin{array}{l}\text { IOP reduction } \\
\text { (from baseline) }\end{array}$ & & $42.5 \%$ & $36.7 \%$ & $40 \%$ & $34.1 \%$ & $33.6 \%$ \\
\hline & Success rate & & $77.2 \%$ & $68.1 \%$ & $72.2 \%$ & $59.1 \%$ & $54.5 \%$ \\
\hline
\end{tabular}

IOP, intraocular pressure; MP-TSCPC, micropulse transscleral cyclophotocoagulation; CW-TSCPC, continuous wave-transscleral cyclophotocoagulation.

Table IV. Variation in the number of topical antiglaucoma medications and in oral acetazolamide use at different time-points after MP-TSCPC and CW-TSCPC.

\begin{tabular}{|c|c|c|c|c|c|c|}
\hline Procedure & Medication & Baseline & 1 month & 3 months & 6 months & 12 months \\
\hline \multirow[t]{2}{*}{ MP-TSCPC } & $\begin{array}{l}\text { Mean number of topical antiglaucoma } \\
\text { medications }\end{array}$ & $2.6 \pm 1$ & $2.3 \pm 1.2$ & $1.7 \pm 1.3$ & $1.9 \pm 1.3$ & $2.1 \pm 1.3$ \\
\hline & Oral acetazolamide users $(\%)$ & $58.3 \%$ & $25 \%$ & $16.7 \%$ & $16.7 \%$ & $20.8 \%$ \\
\hline \multirow[t]{2}{*}{ CW-TSCPC } & $\begin{array}{l}\text { Mean number of topical antiglaucoma } \\
\text { medications }\end{array}$ & $2.8 \pm 0.8$ & $1.7 \pm 1.3$ & $1.4 \pm 1.4$ & $1.7 \pm 0.9$ & $1.9 \pm 1.1$ \\
\hline & Oral acetazolamide users $(\%)$ & $63.6 \%$ & $38 \%$ & $27.2 \%$ & $13.6 \%$ & $27.2 \%$ \\
\hline
\end{tabular}

MP-TSCPC, micropulse transscleral cyclophotocoagulation; CW-TSCPC, continuous wave-transscleral cyclophotocoagulation.

was the lowest at 6 months $(13.6 \%)$, with a subsequent increase at 12 months $(27.2 \%)$. The results are revealed in Table IV and Fig. 2.
Complications. All complications encountered during our study are comparatively listed for both groups in Table V. Complications were more frequent in the CW-TSCPC group. 
A

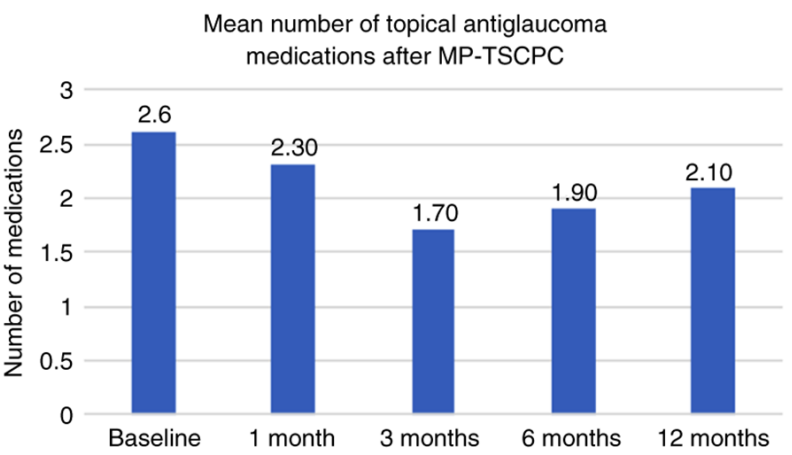

B

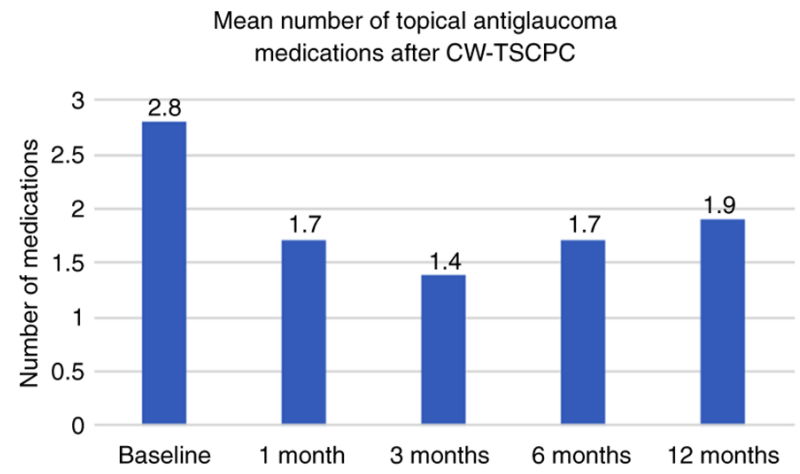

C

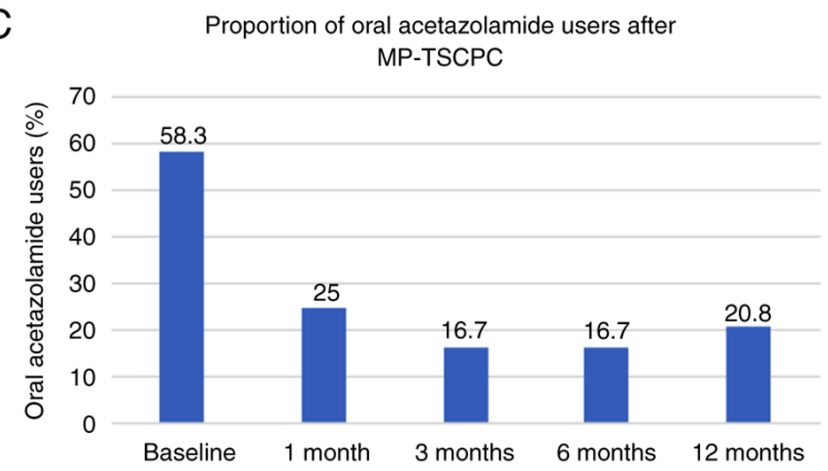

$\mathrm{D}$



Figure 2. Evolution of the number of topical medications and of oral acetazolamide use at different time-points after MP-TSCPC and CW-TSCPC. The four graphs revealed the mean number of topical antiglaucoma medications required per patient at baseline as well as months 1,3,6 and 12 after (A) MP-TSCPC and after (B) CW-TSCPC and the percentage of acetazolamide users at baseline as well as months 1, 3, 6 and 12 for (C) MP-TSCPC and (D) CW-TSCPC. CW-TSCPC, continuous wave transscleral cyclophotocoagulation; MP-TSCPC, micropulse transscleral cyclophotocoagulation.

Moreover, the incidence of important adverse events (worsening of the VA, hypotony, and phthisis bulbi) was, as already stated, significantly greater in the CW-TSCPC group than in the MP-TSCPC group $(\mathrm{P}=0.045)$. VA was at an extremely low level in our cohort at baseline. VA worsened in 8 (36.4) cases in the CW-TSCPC, vs. 4 (16.6\%) in the MP-TSCPC group. Only the VA of 1 patient improved and that occurred in the MP-TSCPC group. The more frequent worsening of VA in the CW-TSCPC group was not statistically significant, but it reached a trend level $(\mathrm{P}=0.1)$, as demonstrated in Table VI. Hypotony was present in 4 cases $(18.2 \%)$ in the CW-TSCPC group and in 2 cases $(8.3 \%)$ in the MP-TSCPC group $(\mathrm{P}=0.41)$. The devastating complication of phthisis bulbi appeared in 2 cases (9.1\%), both in the CW-TSCPC group. Retreatment was necessary in 6 cases in the MP-TSCPC group; 4 cases underwent retreatment 3 months after the first procedure, one case after 4 months, and one case after 6 months. In the CW-TSCPC group, 7 cases were retreated, most of them after 3 months ( 5 cases), one case after 6 months, and one case after 10 months.

\section{Discussion}

NVG is one of the most difficult to treat types of glaucoma. Numerous treatment methods have been used with varying degrees of success. TSCPC is a classic method used to treat NVG, belonging to the broad category of cycloablative procedures. This method has some advantages: i) it is incision-free, and thus, has a very low risk of infection; ii) it is easy to perform (in the operating room or even in an office setting); iii) it has a very short learning curve (compared with trabeculectomy and glaucoma drainage devices); iv) there is no need to stop anticoagulants; v) there is a rapid onset of the effect; and, what is more, vi) it is repeatable (12). The results of our study confirmed that TSCPC can be a safe and reliable method for managing NVG, using both of its variants, MP-TSCPC and CW-TSCPC, each of them with advantages and disadvantages. A successful result was defined as a postprocedural IOP between 5 and $21 \mathrm{~mm} \mathrm{Hg}$ with or without additional medications or an IOP reduction of more than $30 \%$ compared with the baseline. Hypotony, defined as an IOP of $<5 \mathrm{~mm} \mathrm{Hg}$ was considered to be a failure of the treatment. The definition of a successful result is a major problem because it shows great variation among different studies. The majority of other studies defined success as an IOP between 5 and $21 \mathrm{~mm} \mathrm{Hg}$ (13-15); other studies also included a reduction in IOP with at least $30 \%$ from the baseline, as for example in a study by Aquino et al (5), or a reduction with at least $20 \%$ from the baseline IOP, as for example in a study by Grueb et al (16). Our cohort included patients with advanced NVG, with high initial IOP and poor initial VA and therefore it was considered that a reduction of IOP with $20 \%$ would be inefficient in most cases.

Effects on IOP. MP-TSCPC proved to be short-term effective. The success rate was $70.8 \%$ after the first week, $66.6 \%$ after the first month, and $58.3 \%$ after 3 months. The success rate decreased after three months and reached a level of only $29.1 \%$ at 12 months. Numerous studies have reported better results than ours for MP-TSCPC. Tan et al reported a success rate of $80 \%$ after 18 months of follow-up (10), Aquino et al revealed a $75 \%$ success rate after 12 months (5), Preda et al reported a $65.63 \%$ success rate at 18 months (17), and Zaarour et al showed a $73.3 \%$ success rate at 12 months (18), but all of these studies included patients with different forms 
Table V. Complications encountered after TSCPC.

\begin{tabular}{lll}
\hline Complications & Micropulse TSCPC & Continuous wave TSCPC \\
\hline Important adverse events & & 0.045 \\
Worsening of visual acuity & $4(16.6 \%)$ & $8(36.4 \%)$ \\
Hypotony & $2(8.3 \%)$ & $4(18.2 \%)$ \\
Phtisis bulbi & $0(0 \%)$ & $2(9.1 \%)$ \\
Other adverse events & & 0.41 \\
Prolonged inflammation & $1(4.1 \%)$ & $3(13.6 \%)$ \\
Choroidal detachment & $1(4.1 \%)$ & $4(18.2 \%)$ \\
Postoperative intraocular pressure spike & $4(16.6 \%)$ & $4(18.2 \%)$ \\
Retinal detachment & $0(0 \%)$ & $3(13.6 \%)$ \\
Hyphema & $3(12.5 \%)$ & $2(9.1 \%)$ \\
Neurotrophic keratitits & $0(0 \%)$ & $1(4.5 \%)$ \\
Intravitreal hemorrhage & $0(0 \%)$ & $3(13.6 \%)$ \\
\hline
\end{tabular}

Important adverse events include worsening of visual acuity, hypotony and phtisis bulbi. TSCPC, transscleral cyclophotocoagulation.

Table VI. Changes in VA after TSCPC.

\begin{tabular}{lcc}
\hline Evolution of best-corrected VA & Micropulse TSCPC N (\%) & Continuous wave TSCPC N (\%) $^{\text {P-value }}$ \\
\hline Worsened & $4(16.6 \%)$ & $8-(36.4 \%)$ \\
Unchanged & $19(79.2 \%)$ & $14-(63.6 \%)$ \\
Improved & $1(4.2 \%)$ & 0.10 \\
\hline
\end{tabular}

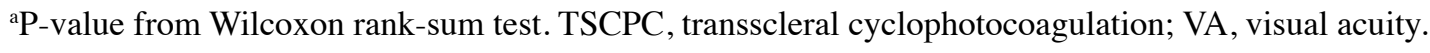

of refractory glaucoma, not only NVG. Studies on cohorts with NVG revealed slightly poorer outcomes, but some reported improved results, for example, Wong et al reported a $26 \%$ success rate at 12 months and Souissi et al revealed a $35 \%$ success rate at 9 months $(19,20)$. A total of 2 possible explanations were identified for this difference. Firstly, our cohort was composed of patients with advanced NVG (extremely low VA, multiple surgeries) and, secondly, the laser settings, which were not at the highest possible level of energy, may have contributed to inadequate control of IOP in the long term. It is possible that to achieve a satisfactory IOP control in patients with NVG, but an increase in the duration of laser delivery may be necessary. A study by Williams et al revealed improved results with a $74.7 \%$ success rate at 3 months with a longer time interval of laser application, of up to $360 \mathrm{sec}$, while using the same power (11). CW-TSCPC exhibited a more constant effect during the follow-up period. The success rate was $77.2 \%$ after the first week, $68.1 \%$ after the first month, $72.2 \%$ at 3 months, and $54.5 \%$ at 12 months, results that are similar to those of other studies. Singh et al (21) reported a success rate of $70 \%$ and Grueb et al (16), a 36.7\% success rate, with different periods of follow-up.

Effect on antiglaucoma medication. Both methods resulted in a decrease in the number of topical antiglaucoma medications in the short term, which is similar to the results reported by other studies. MP-TSCPC had the most important reduction in medication number at 3 months, with the mean number of medications decreasing from a baseline of $2.6 \pm 1$ to $1.7 \pm 1.3$, which was followed by a slight increase to $2.1 \pm 1.3$ at 12 months. CW-TSCPC had similar results at 3 months, a decrease from $2.8 \pm 0.8$ at baseline to $1.4 \pm 1.4$ at 3 months, but the results tended to be more stable in time reaching $1.9 \pm 1.1$ at 12 months. The possibility to stop the carbonic anhydrase inhibitor was an important endpoint of the study and both methods proved effective in reaching this goal. The number of patients requiring oral acetazolamide decreased after both procedures: from $58.3 \%$ of patients at baseline to $20.8 \%$ at 12 months in the MP-TSCPC group and from $63.6 \%$ of patients at baseline to $27.2 \%$ at 12 months in the CW-TSCPC group. Numerous studies have reported a decrease in the number of antiglaucoma medications similar to our results $(1,5,18,22)$.

Complications. Postoperative complications appeared in both groups, but the incidence was higher in the CW-TSCPC group. There were 15 complications in 9 patients $(37.5 \%)$ in the MP-TSCPC group and 34 complications in 15 patients (68.2\%) in the CW-TSCPC group. Potential complications in CW-TSCPC are considered to be secondary to damage induced to the surrounding tissues by the thermal effect of the laser. In MP-TSCPC the pulsatile pattern of the laser 
energy delivery prevents excessive heating of the collateral tissues and reduces the rate of complications (6). It is difficult to establish a cause-and-effect relationship between TSCPC and the complications, because some may appear as complications of the initial disease as for example, tractional retinal detachment and intravitreal hemorrhages in diabetic retinopathy, as well as late hyphema (2 cases in the MP-TSCPC group and one case in the CW-TSCPC appeared more than 3 months after the procedure). The most frequent complication was a decrease in VA; this occurred in $16.6 \%$ of cases in the MP-TSCPC group and in $36.4 \%$ in the CW-TSCPC group. In other studies worsening of the VA varies from 0 to $55.2 \%$ of the cases (23-25). However, it is difficult to compare our results with those of other studies, because in our study the baseline BCVA was already extremely poor (CF or less). Herein, although a higher incidence of VA decline was reported after MP-TSCPC than in other studies such as Aquino et al who revealed a $4 \%$ deterioration of BCVA and Elhefney et al and Lee et al who revealed no decline in BCVA, the fact that some of the cases probably had a decrease in VA as a result of the evolution of the disease, and not as a result of the procedure itself, must be taken into account $(5,26,27)$. Even though the difference between the two groups was not statistically significant, it reached the trend level, and therefore MP-TSCPC is considered to be safer than CW-TSCPC in what post-procedural BCVA is concerned.

Another serious complication that was identified in our study was ocular hypotony. It appeared in 2 patients $(8.3 \%$ of cases) in the MP-TSCPC group and in 4 patients $(18.2 \%$ of cases) in the CW-TSCPC group. Unfortunately, two of these cases finally progressed to phthisis bulbi (9.1\% of cases). In other studies, some reported an incidence of hypotony similar to ours in the case of CW-TSCPC, including Iliev and Gerber (15) with 17.6\%, and Walland (28) with $18 \%$, while some reported a higher incidence such as the study by Nabili and Kirkness (22), but there were also studies reporting a much lower incidence including studies by Vernon et al (25) and Schlote et al (8). Studies with a similar or higher incidence of hypotony included a greater percentage of patients with NVG, whereas the other ones had few or no patients with NVG. This may be explained by the fact that eyes with NVG have a disproportionate outflow resistance, while the aqueous humor production is already damaged by ischemia. As such, any cyclodestructive procedure, even a mild one, as is the case with MP-TSCPC, can disturb the balance between outflow resistance and the aqueous production, resulting in hypotony (22). Our study revealed that, with regard to adverse effects (i.e., the decrease in VA, hypotony, and phthisis bulbi), their higher occurrence rate in the CW-TSCPC group vs. in the MP-TSCPC group was statistically significant $(\mathrm{P}=0.045)$.

Limitations. The most important limitation of our study resides in the small sample size for each group (24 and 22 cases, respectively, for MP-TSCPC and CW-TSCPC). Another limitation emerges from the retrospective nature of the study and from the fact that not all the data were available for all the patients. However, the patients were observed concurrently by the authors and followed the same visit schedule and, what is more, the study had a 12-month follow-up on 46 of the 51, or $90 \%$, of the patients who underwent the intervention. Our center is a tertiary care center, and thus the postoperative visits of some of the patients took place in their primary care center. A lot of effort was made to retrieve the data from those primary care centers, however, it cannot be certain that the accuracy of the data is the same.

In conclusion, both methods, MP-TSCPC and CW-TSCPC, could successfully manage NVG. CW-TSCPC exhibited higher IOP control in the long term (which did not reach statistical significance), but a significantly lower safety profile. MP-TSCPC was revealed to be safer, but its efficacy may decline after three months. Patients with advanced NVG may require higher laser energy or longer application time.

\section{Acknowledgements}

Not applicable.

\section{Funding}

No funding was received.

\section{Availability of data and materials}

The datasets used and/or analyzed during the current study are available from the corresponding author on reasonable request.

\section{Authors' contributions}

MZ, DCB, FB, MB, IRB and FV conceived and designed the study. MZ performed the surgical procedures. MZ, OMD, EAD, ACS and IP performed the acquisition of data during the follow-up visits. MZ, DCB, IRB, OMD, EAD, IP, ACS and $\mathrm{FV}$ participated in the analysis and interpretation of the data. FV performed the statistical analysis. MZ, FB and MB drafted the manuscript. MZ, DCB, FB, MB and IRB critically reviewed the manuscript. $\mathrm{MZ}$ and OMD confirm the authenticity of all the raw data. All authors read and approved the final manuscript.

\section{Ethics approval and consent to participate}

The study was approved by the institutional review board of 'Dr. Carol Davila' Central Military Emergency University Hospital (Bucharest, Romania) and followed the principles of the Declaration of Helsinki.

\section{Patient consent for publication}

Not applicable.

\section{Competing interests}

The authors declare that they have no competing interests.

\section{References}

1. Fong AW, Lee GA, O'Rourke P and Thomas R: Management of neovascular glaucoma with transscleral cyclophotocoagulation with diode laser alone versus combination transscleral cyclophotocoagulation with diode laser and intravitreal bevacizumab. Clin Exp Ophthalmol 39: 318-323, 2011. 
2. Sivak-Callcott JA, O'Day DM, Gass JD and Tsai JC: Evidence-based recommendations for the diagnosis and treatment of neovascular glaucoma. Ophthalmology 108: 1767-1777, 1800,2001

3. Ndulue JK, Rahmatnejad K, Sanvicente C, Wizov SS and Moster MR: Evolution of cyclophotocoagulation. J Ophthalmic Vis Res 13: 55-61, 2018

4. Burgess SE, Silverman RH, Coleman DJ, Yablonski ME,Lizzi FL, Driller J, Rosado A and Dennis PH Jr: Treatment of glaucoma with high-intensity focused ultrasound. Ophthalmology 93: 831-838, 1986.

5. Aquino MC, Barton K, Tan AM, Sng C, Li X, Loon SC and Chew PT: Micropulse versus continuous wave transscleral diode cyclophotocoagulation in refractory glaucoma: A randomized exploratory study. Clin Exp Ophthalmol 43: 40-46, 2015.

6. Ma A, Yu SWY and Wong JKW: Micropulse laser for the treatment of glaucoma: A literature review. Surv Ophthalmol 64: 486-497, 2019.

7. Pastor SA, Singh K, Lee DA, Juzych MS, Lin SC, Netland PA and Nguyen NT: Cyclophotocoagulation: A report by the American Academy of Ophthalmology. Ophthalmology 108: 2130-2138, 2001.

8. Schlote T, Derse M, Rassmann K, Nicaeus T, Dietz K and Thiel HJ: Efficacy and safety of contact transscleral diode laser cyclophotocoagulation for advanced glaucoma. J Glaucoma 10: 294-301, 2001.

9. Amoozgar B, Phan EN, Lin SC and Han Y: Update on ciliary body laser procedures. Curr Opin Ophthalmol 28: 181-186, 2017.

10. Tan AM, Chockalingam M, Aquino MC, Lim ZI, See JL and Chew PT: Micropulse transscleral diode laser cyclophotocoagulation in the treatment of refractory glaucoma. Clin Exp Ophthalmol 38: 266-272, 2010

11. Williams AL, Moster MR, Rahmatnejad K, Resende AF Horan T, Reynolds M, Yung E, Abramowitz B, Kuchar S and Waisbourd M: Clinical efficacy and safety profile of micropulse transscleral cyclophotocoagulation in refractory glaucoma. J Glaucoma 27: 445-449, 2018 .

12. Stanca HT, Munteanu M, Jianu DC, Motoc AGM, Tăbăcaru B, Stanca S, Ungureanu E, Boruga VM and Preda MA: New perspectives in the use of laser diode transscleral cyclophotocoagulation. A prospective single center observational cohort study. Rom J Morphol Embryol 59: 869-872, 2018.

13. Ramli N, Htoon HM, Ho CL, Aung T and Perera S: Risk factors for hypotony after transscleral diode cyclophotocoagulation. J Glaucoma 21: 169-173, 2012.

14. Murphy CC, Burnett CA, Spry PG, Broadway DC and Diamond JP: A two centre study of the dose-response relation for transscleral diode laser cyclophotocoagulation in refractory glaucoma. Br J Ophthalmol 87: 1252-1257, 2003.

15. Iliev ME and Gerber S: Long-term outcome of trans-scleral diode laser cyclophotocoagulation in refractory glaucoma. Br J Ophthalmol 91: 1631-1635, 2007.
16. Grueb M, Rohrbach JM, Bartz-Schmidt KU and Schlote T: Transscleral diode laser cyclophotocoagulation as primary and secondary surgical treatment in primary open-angle and pseudoexfoliatve glaucoma. Long-term clinical outcomes. Graefes Arch Clin Exp Ophthalmol 244: 1293-1299, 2006.

17. Preda MA, Karancsi OL, Munteanu M and Stanca HT: Clinical outcomes of micropulse transscleral cyclophotocoagulation in refractory glaucoma-18 months follow-up. Lasers Med Sci 35: 1487-1491, 2020.

18. Zaarour K, Abdelmassih Y, Arej N, Cherfan G, Tomey KF and Khoueir Z: Outcomes of micropulse transscleral cyclophotocoagulation in uncontrolled glaucoma patients. J Glaucoma 28: 270-275, 2019

19. Wong KYT, Aquino CM, Macasaet AM, Suwandono ME, Chew PTK and Koh VTC: MP3 plus: A modified micropulse transscleral cyclophototherapy technique for the treatment of refractory glaucoma. J Glaucoma 29: 264-270, 2020.

20. Souissi S, Baudouin C, Labbé A and Hamard P: Micropulse transscleral cyclophotocoagulation using a standard protocol in patients with refractory glaucoma naive of cyclodestruction. Eur J Ophthalmol 31: 112-119, 2021.

21. Singh K, Jain D and Veerwal V: Diode laser cyclophotocoagulation in Indian eyes: Efficacy and safety. Int Ophthalmol 37: 79-84, 2017.

22. Nabili S and Kirkness CM: Trans-scleral diode laser cyclophoto-coagulation in the treatment of diabetic neovascular glaucoma. Eye (Lond) 18: 352-356, 2004.

23. Ishida K: Update on results and complications of cyclophotocoagulation. Curr Opin Ophthalmol 24: 102-110, 2013.

24. Ocakoglu O, Arslan OS and Kayiran A: Diode laser transscleral cyclophotocoagulation for the treatment of refractory glaucoma after penetrating keratoplasty. Curr Eye Res 30: 569-574, 2005.

25. Vernon SA, Koppens JM, Menon GJ and Negi AK: Diode laser cycloablation in adult glaucoma: Long-term results of a standard protocol and review of current literature. Clin Exp Ophthalmol 34: 411-420, 2006.

26. Elhefney EM, Mokbel TH, Hagras SM, AlNagdy AA, Ellayeh AA, Mohsen TA and Gaafar WM: Micropulsed diode laser cyclophotocoagulation in recurrent pediatric glaucoma. Eur J Ophthalmol 30: 1149-1155, 2020.

27. Lee JH,Shi Y,Amoozgar B, Aderman C, De Alba Campomanes A, Lin S and Han Y: Outcome of micropulse laser transscleral cyclophotocoagulation on pediatric versus adult glaucoma patients. J Glaucoma 26: 936-939, 2017.

28. Walland MJ: Diode laser cyclophotocoagulation: Longer term follow up of a standardized treatment protocol. Clin Exp Ophthalmol 28: 263-267, 2000.

This work is licensed under a Creative Commons Attribution-NonCommercial-NoDerivatives 4.0 International (CC BY-NC-ND 4.0) License. 